\title{
Direct detection of temperature maps on electronic devices surface by using an infrared radiometric microscope
}

by: PICA S. and SCARPETTA G.

University of Naples, Department of Electronics, Via Claudio 21, 80125, Naples (Italy).

\begin{abstract}
In this paper the advantages of using infrared radiometry for temperature measurements on semiconductor devices are presented. The choice of using a radiometric microscope with an automatic scanning and elaboration system, instead of thermal imaging, is discussed. Some peculiar problems of thermal measurements on electronic devices, and their solutions are also presented. Finally, preliminary results of our system are shown, consisting in dynamic temperature maps obtained on two different devices, with a brief discussion on their different thermal instability.
\end{abstract}

\section{Introduction}

The infrared radiometry is a contactless technique for temperature measurements. This is an important feature, because it allows not to alter the electric and thermal environment of the electronic device in working conditions. Another important characteristic of this technique is the possibility, with a microscopic configuration, to obtain a measuring spot of very little size, as required in microelectronics. In fact, while the importance of temperature measurements in semiconductor devices is increasing, the dimensions of technological structures are decreasing. This is especially true for power and integratedpower (smart-power) devices which dissipate a large thermal power.

For these devices a problem of thermal stability also exists, consisting in a possible uneven current distribution in the available area with a consequent non uniform temperature distribution, that in some cases can lead to the destruction of the device. This effect determines a limitation of the effective safe operating area (SOA ). To this point, the detection of dynamic temperature maps is very important to follow the transient evolution of the temperature distribution, in order to better investigate the physical and technological factors that determine the thermal instability .

\section{A system for thermal mapping on electronic devices}

\subsection{An overview on thermal measurement systems on electronic devices}

In the last twenty years a great effort has been dedicated to develop an high resolution system of infrared microscopy suitable to extend the thermal inspection, already realized for printed board, to integrated circuits [ 1 ]. As a result of this effort, many commercial instruments have been proposed. These are typically constituted of an infrared sensor, an optical system for collecting and focusing the infrared radiation, a movable stage, that allows the scanning of the target, and a control and elaboration system to produce the temperature output in the desired form [ 2 ].

The infrared detectors typically used are realized in indium-antimonide, and work in an infrared band between 2 and $5 \mu \mathrm{m}$. This choice results to be the better trade-off between 
http://dx.doi.org/10.21611/qirt.1994.005

the thermal emission of the heated device, the resolution of the optical apparatus, and the available technology of sensors.

All the radiometric systems are based on the self-emitted infrared radiance from target, but true-temperature mapping can be accomplished only when target emissivity is known. A thermal map, however, contains points, of a surface on which there are materials of different emissivity. To solve this problem, an infrared system must include a temperaturecontrolled heated sub stage, to perform an emissivity computation and correction routine.

The first step in this routine is an operating calibration in which the device to be mapped is heated at one or two controlled temperatures, and scanned in an unpowered mode. The radiance scan data is stored and used to compute the emissivity of the target, point by point. When the device is subsequently powered up, the resulting radiance image is automatically corrected by the stored emissivity matrix, and a temperature map results.

Starting from 1980 many commercial imaging systems have appeared, including specially adapted personal computers and display terminals, thus creating thermal mappers that can perform various functions, to acquire, display and process quantitative thermal images.

In some of this systems, the scansion is performed by the optical systems, through a moving-mirror apparatus, in some others there is a motorized stage to move the target.

\subsection{A bidimensional microscopic system}

Our system is based on an infrared radiometric microscope, and a scanning system to obtain a bidimensional map. The control of the scanning, and the different steps of the acquisition and elaboration routine are controlled by a PC through a home-made software.

With respect to the closed-box thermal imaging system as those we have described before, this system requires certainly a longer and harder work to obtain a temperature map, in addition to the work that has required its realization and optimization, nevertheless this system presents some advantages for the features of this kind of measurements.

First of all, this is certainly a low cost system. In addition it allows a better spatial resolution on wider areas, and has a shorter response time with respect to the automatic scanning systems, which is important to follow in detail fast thermal transients. However, the greater advantage of a self-made software is the greater flexibility, which is important to overcome some problems that we will discuss in the following.

In a microscopic configuration, some additive errors are present, such as Narcissus effect and other background reflections, due to the small working distance between the objective and the target. We have developed a correction routine, based on a black-body model at ambient temperature, and a recursive software elaboration fastly converging.

Moreover, it is important to get the radiance from the same points in the two scanning (powered and the unpowered), in order to correctly compensate each point for its emissivity. This goal may be not easy to obtain, because between the two scansions, the device and its sub stage, that is a water cooled heat sink, are subjected to thermal and mechanical stress; so, for example, the different temperature conditions determine different thermal expansion of the materials. For this reason, it is fundamental to identify an absolute position reference on the chip layout. To this extent, we use a crosscorrelation technique between the two maps. Another goal of the software is to cut off the high-frequency noise of the infrared detector, by realizing a low-pass filtering.

For dynamic measurements, a further difficulty regards the low signal-to-noise ratio, due to the fact that the signal directly comes from the detector through a preamplifier, and is not mechanically chopped, as in the static mode. To solve this problem an averaging of the signal on many periods is needed, which rejects the non correlated noise.

Finally, a calibration procedure is needed to validate the results, using an independent reference on the device, namely a TSEP (temperature sensitive electrical parameter). 


\subsection{Hardware and software description}

The measurement system is centered on the infrared microscope. Under the microscope's objective is the mechanical section, consisting in a water cooled heat sink, and in different stages for $x-y$ translation, rotation, and tilt regulation of the device. The heat sink works also as heating sub stage in the emissivity matrix determination. The device to inspect is placed on the heat sink, and is biased in the DC or in the pulsed mode by the electrical section. Main characteristics of radiometer and heat sink/heater are reported in Table 1.

The radiance of the target is sent to a data-acquisition system, together with the outputs of the $x-y$ position sensors, and with the heat sink temperature.

The different steps of the measurement routine are controlled by a specific software, that manages the data acquisition and the elaboration needed to obtain the final output.

In figure 1 it is displayed an example of the results that we can obtain in the two phases of calibration and static measurement. The marked portion of the device has been scanned at the maximum spatial resolution allowable $(10 \mu \mathrm{m})$. Note how the strong variations that are present in the emissivity map, are well compensated in the temperature map.

\section{Experimental results}

In figure 2 and figure 3 some temperature maps are reported, at the time instants marked, for two power bipolar transistors, with rather different technology, in particular the second one (named BOO8) has smaller dimensions in the elementary cell that is replied on the whole surface (cellular structure).

The devices have been biased in an equivalent electrical condition, keeping into account the different area (power density $=8 \mathrm{~W} / \mathrm{mm}^{3}$ ), such to bring them in a current crowding condition. This determines a greater local heating, that can degenerate in a stable hot spot.

A different thermal behavior is observed. In particular, the device with a cellular geometry presents a faster onset of the hot spot growth, and a smaller area of the same, and this indicates a greater thermal instability. For both devices the hot spot zone is located near to the pad that brings the greater current [ 3 ].

A preliminary interpretation of this different behavior regards the differences in the layout. In particular, if we regard the device as constituted by a number of elementary cells placed in a parallel network, the smaller dimensions in the elementary cell correspond to a smaller value in the electrical resistance associated to the cell. Because this resistance play a stabilizing-role in an electro-thermal feedback set up, a larger instability in the second device is expected [ 4 ].

Anyway, a quantitative interpretation of the big amount of data that we obtain with this system, can be achieved only with the help of an electro-thermal model of the device. A guideline in the design of this model can be the discrete multi-cell representation introduced before, that suggest a circuital model of the electrical and thermal paths.

The main difficulties in realizing this model, are the correct estimation of the value of various parameters to attribute to the single cell, and the correct modeling of the interactions between the electrical and thermal parameters.

\section{Conclusions}

The development of an automatic system to produce static and dynamic temperature maps on electronic structures has been presented, discussing about its importance and 
http://dx.doi.org/10.21611/qirt.1994.005

about the problems connected with this realization. Some interesting results have also been presented on the thermal instability of power devices. In the next future we are going to improve this system, in terms of a wider automatization, and we are going to inspect in the theoretical interpretation of the data collected.

\section{Acknowledgments}

The authors wish to thank Mr. Antonio Zecchino for the design and the realization of the mechanical parts of the set up.

\section{REFERENCES}

( 1 ] KAPLAN (H.), IR imaging's latest crop, Photonics Spectra, Sept. 1984, The Optical Publishing Co.

( 2 ] ASTHEMEIR. (R. W.), Handbook of infrared measurements. B. E. C., 1983

( 3 ] HOWER (P. L.), BLACKBURN (D. L.), OETTINGER (F.F.), RUBIN (S.), Stable hot spots and second breakdown in power transistors, IEEE PESC, 1976, pp. 234-246

( 4 ] GHANDI (S. K.), Semiconductor Power Devices, John Wiley \& Sons, New York, 1977

\section{Radiometer : \\ Temperature Resolution: $.0 .5^{\circ} \mathrm{C}$ \\ Temperature Range: $0{ }^{\circ} \mathrm{C}$ to $350^{\circ} \mathrm{C}$ \\ Spot Size: $7.6 \mu \mathrm{m}$ \\ Visual Field of View: $250 \mu \mathrm{m}$ \\ Working Distance: $2.5 \mathrm{~mm}$ \\ Detector Type: Indium-Antimonide \\ Spectral Range: 1.8 to $5.5 \mu \mathrm{m}$ \\ Kesponse Time: $\bar{\delta} \mu \mathrm{sec}$}

\section{Heatsink / Heater :}

Thermal resistance between device junction and heatsink (for typical case and device):.. 1.7 to $2.5^{\circ} \mathrm{C} / \mathrm{W}$

Maximum power removable as heatsink:... (at $25^{\circ} \mathrm{C}$ ) up to $200 \mathrm{~W}$

Maximum temperature as heater.....200 ${ }^{\circ} \mathrm{C}$

Table 1. - Main specifications of radiometer and of heatsink/heater

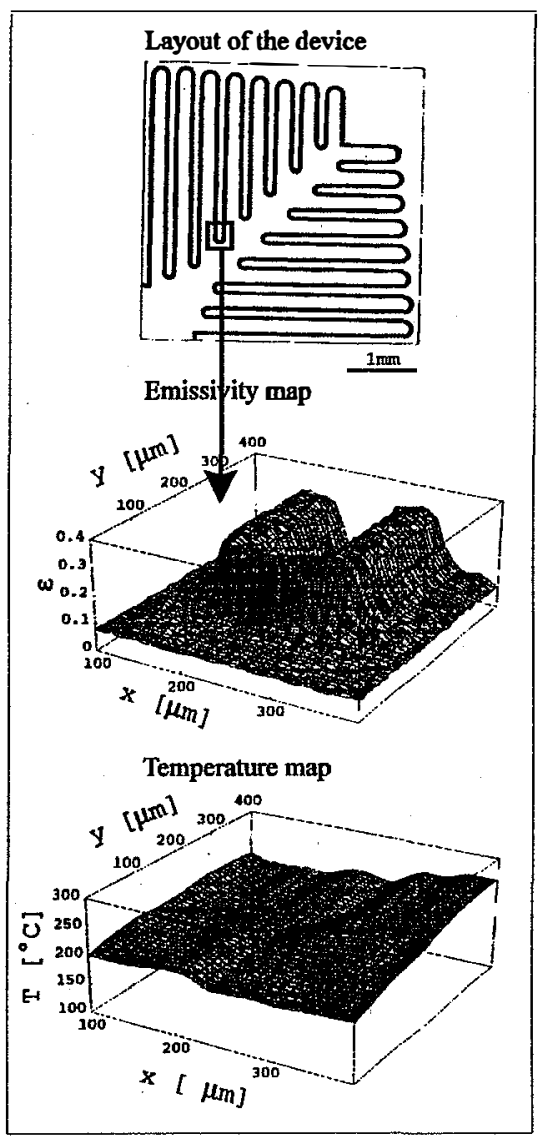

Fig. 1. - High resolution emissivity and temperature maps 

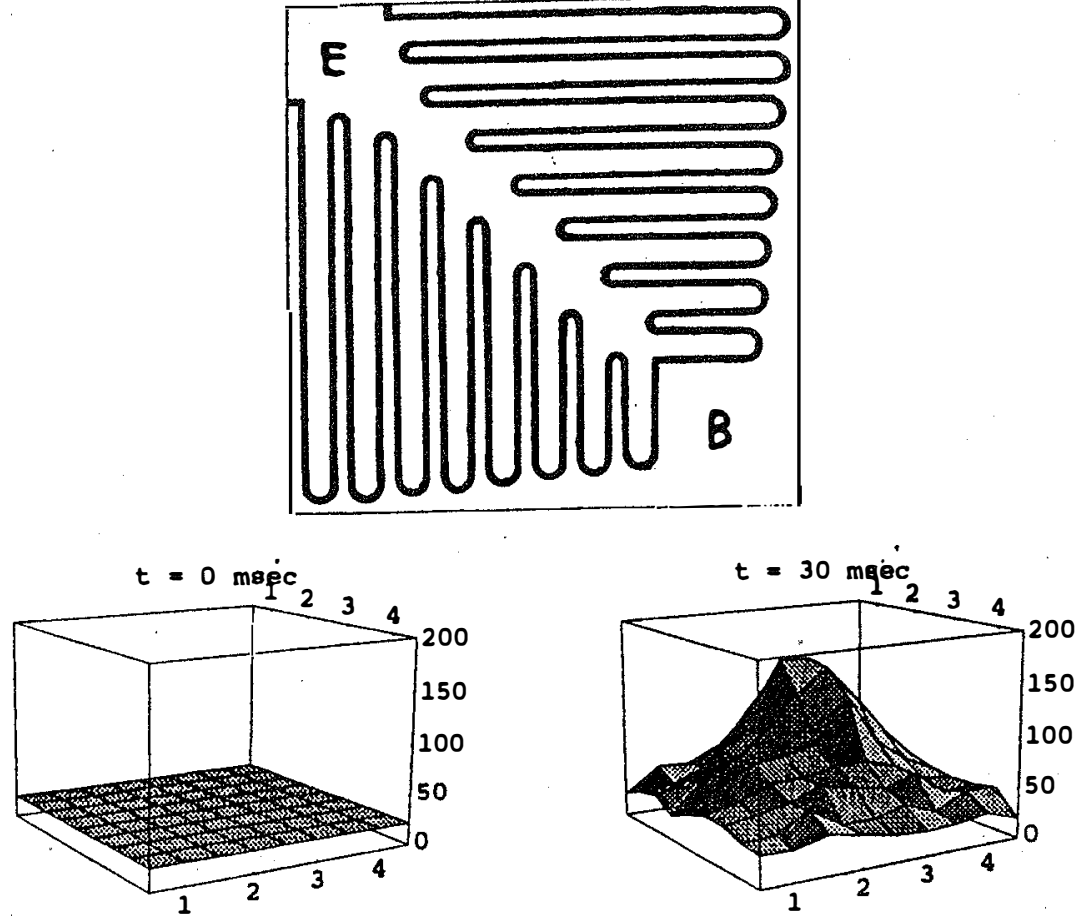

$t=10 \mathrm{mgec}$

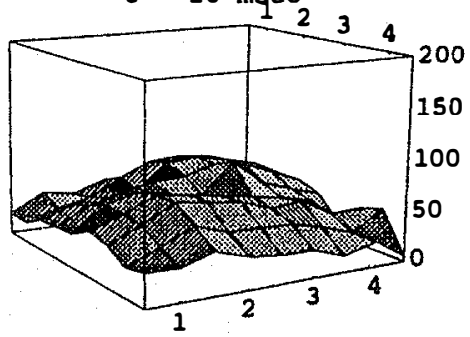

$t=40 \mathrm{mgec}$

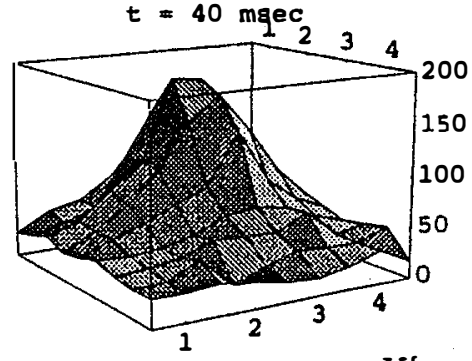

$t=20 \mathrm{mgec}$
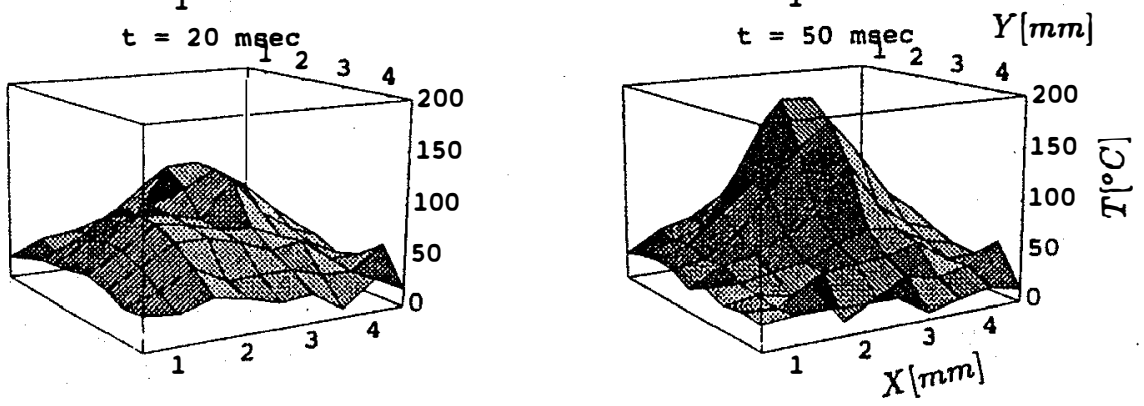

Fig.2. - Layout of BJT-BUY69 and temperature maps at the times marked 

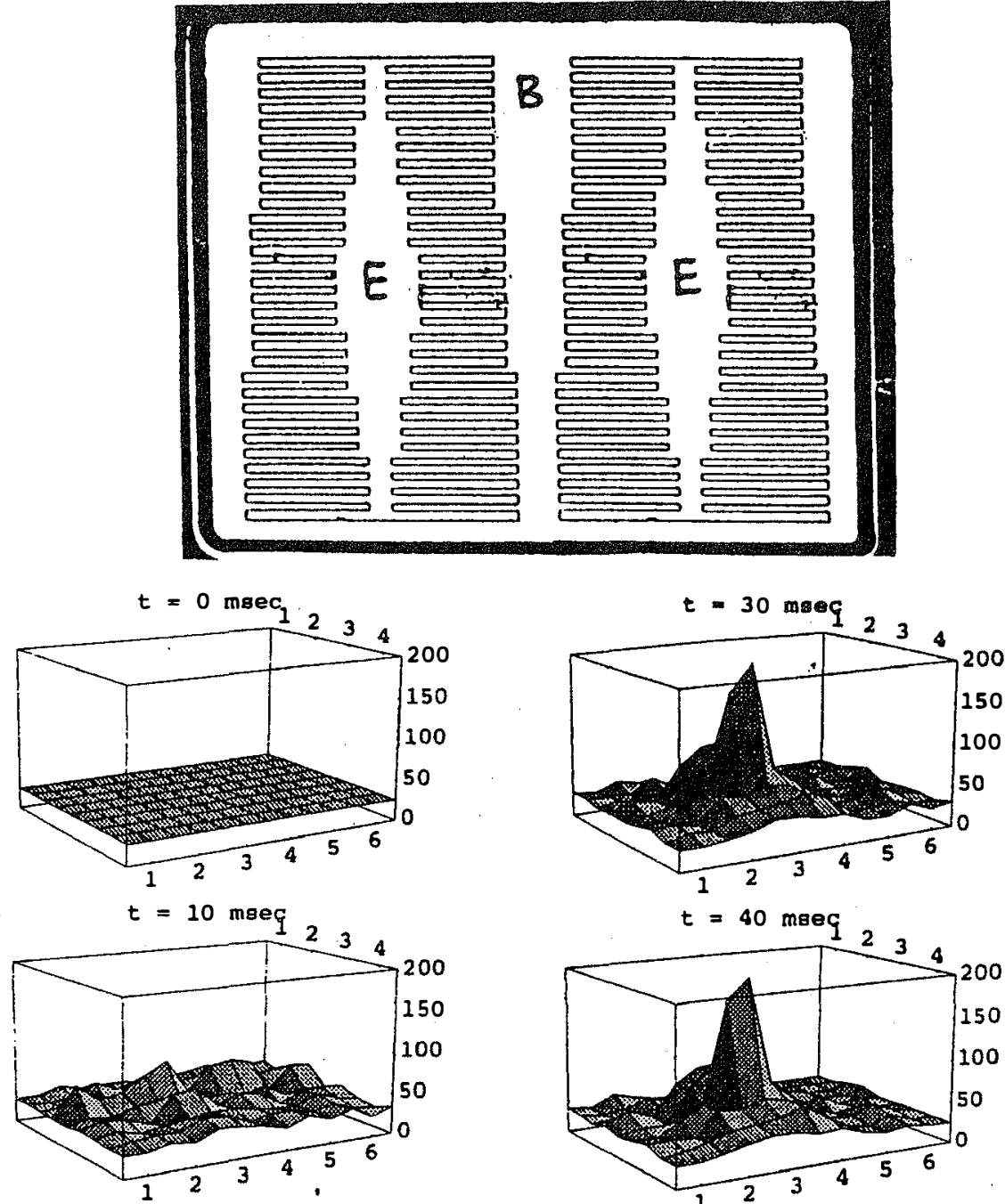

$t=40 \mathrm{meec}$

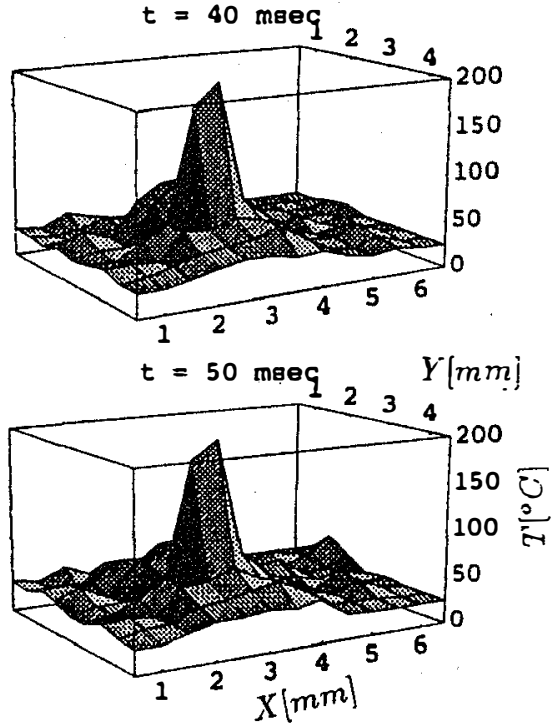

Fig.3. - Layout of BJT-B008 and temperature maps at the times marked 